\section{In-hospital VTE risk-many patients do not receive appropriate prophylaxis}

Venous thromboembolism (VTE) and complications due to VTE are a major cause of morbidity and mortality in hospitalized patients. The multinational ENDORSE study examined the prevalence of risk factors for VTE and the use of VTE prophylaxis in patients receiving acute hospital care. The researchers found that, although approximately half of all patients were at risk for VTE, many patients, and in particular medical as opposed to surgical patients, did not receive appropriate prophylaxis.

The study enrolled 68,183 patients from 358 hospitals in 32 countries, who were assessed for VTE risk according to the 2004 guidelines from the American College of Chest Physicians (ACCP). The most common pre-admission risk factors for VTE were obesity in surgical patients, and chronic pulmonary disease or heart failure in medical patients. The most common in-hospital risk factors were immobilization, and admission to intensive or critical care. ACCP-recommended prophylaxis was received by $50.2 \%$ of all at-risk patients $(58.5 \%$ of at-risk surgical patients and $39.5 \%$ of atrisk medical patients). Between-country variation was considerable, with $\sim 80 \%$ of at-risk patients receiving recommended prophylaxis in Germany and Switzerland, compared with $\sim 60 \%$ in the US and UK, $<30 \%$ in Russia, India, Pakistan and Venezuela, and $<1 \%$ in Bangladesh and Thailand.

The authors conclude that prophylaxis for VTE, the most common preventable cause of in-hospital death, is underused, particularly for medical patients. Hospital-wide strategies for the assessment of VTE risk are required, to ensure that at-risk patients receive appropriate treatment.

Original article Cohen AT et al. (2008) Venous thromboembolism risk and prophylaxis in the acute hospital care setting (ENDORSE study): a multinational crosssectional study. Lancet 371: 387-394

\section{Certain definitions of metabolic syndrome might better predict new vascular events}

Different definitions exist for metabolic syndrome, but it is unknown whether the criteria used to diagnose the syndrome affect a patient's outcome. Wassink et al. investigated this issue with regard to cardiovascular events by conducting a prospective study. They concluded that patients with clinically manifest vascular disease and metabolic syndrome as defined by the National Cholesterol Education Program (NCEP) criteria or the revised NCEP (NCEP-R) criteria might benefit from more-aggressive treatment of cardiovascular risk factors.

In all, 3,196 consecutive patients with either a history or a recent diagnosis of atherosclerotic vascular disease were recruited from the University Medical Centre Utrecht from January 1996 to March 2005. The patients were assessed for the presence of metabolic syndrome according to the NCEP criteria, the NCEP-R criteria, and the International Diabetes Federation (IDF) criteria ( $n=1,362$ [43\%], 1,601 [50\%] and 1,375 [43\%], respectively). Patients who met the NCEP and NCEP-R criteria had notably increased risks of new vascular events (hazard ratio 1.50, 95\% Cl 1.22-1.85, after adjustment for age and sex) and mortality (hazard ratio $1.43,95 \% \mathrm{Cl} 1.15-1.78$, after adjustment for age and sex). This risk was irrespective of the presence of type 2 diabetes mellitus and the site of clinical manifestation of atherosclerosis. In addition, in patients who were at recommended levels of systolic blood pressure $(<140 \mathrm{mmHg})$ or LDL cholesterol $(<2.5 \mathrm{mmol} / \mathrm{l})$ according to current guidelines, presence of NCEP-R-defined metabolic syndrome pointed to increased cardiovascular risk.

\footnotetext{
Original article Wassink AMJ et al. (2008) Metabolic syndrome and the risk of new vascular events and allcause mortality in patients with coronary artery disease, cerebrovascular disease, peripheral arterial disease or abdominal aortic aneurysm. Eur Heart J 29: 213-223
} 